\title{
C-Reactive Protein for the Enhanced Evaluation of the Systemic Inflammatory Response Syndrome (SIRS)
}

\author{
Gitta Pancer ${ }^{1,2}$, Ester Engelman ${ }^{1,2}$, Farhana Hoque ${ }^{1,3}$, Mohammed Alam ${ }^{1}$, James Rucinski ${ }^{1}$ and \\ Larry H. Bernstein*,1
}

${ }^{I}$ New York Methodist Hospital, Brooklyn, New York, USA

${ }^{2}$ Touro College, Brooklyn, New York, USA

${ }^{3}$ Midwood High School, Brooklyn, New York, USA

\begin{abstract}
Introduction: An elevation in the CRP may provide an early indication of developing SIRS with progression to sepsis that is more sensitive than the standard clinical criteria of fever (or hypothermia), tachypnea, tachycardia, and leukocytosis with neutrophilia (or neutropenia). The problem of false positive rate for SIRS resides mainly in the common occurance of tachypnea and tachycardia in presenting patients, and in the confounding presence of neutrophilia without reference to good measures of a left shift. The objective of this study was to investigate how using CRP as a marker to confirm the presence of early sepsis might reduce the false positive rate inherent in the defining SIRS criteria.
\end{abstract}

Materials and Methods: One hundred sixty eight patients with leukocytosis greater than 12,000 associated with a high absolute neutrophil count were studied. Those that met the inclusion criteria were analyzed for CRP response.

Results: A linear correlation between CRP elevation and the defining criteria for SIRS was found except there was no correlation with absolute neutrophil count.

Conclusion: CRP is a sensitive indicator of SIRS with advantages over neutrophilia for detecting early sepsis.

Keywords: Systemic inflammatory response, C-reactive protein, early sepsis, SIRS criteria.

\section{INTRODUCTION}

This study is a clinical evaluation of whether using Creactive protein, a test that has been sparingly used in recent years [1], adds a justified benefit in accurately and rapidly identifying acute infection. The test has gained renewed interest as a result of the extensive studies of its relationship to the triad of obesity, insulin resistance, and type 2 diabetes mellitus, with a long term relationship to cardiovascular disease, peripheral vascular disease, stroke, sleep apnea, and hyperlipidemia, referred to as metabolic syndrome. This renewed interest is based on the epidemiological evidence that a normal CRP range is under $5 \mathrm{mg} / \mathrm{L}$ and a mild elevation at $5-10 \mathrm{mg} / \mathrm{L}$ carries a 3.3 times relative risk of coronary vascular disease than an unaffected population. Moreover, recent work shows efficacy of a statin in lowering the risk [2], [Jupiter trial]). On the other hand, we are concerned with the observation of rapidly rising levels of CRP in the patient who presents with an acute bacterial infection and may require antibiotic dosing within 4-6 hours, and transfer to medical intensive care.

The Systematic Inflammatory Response Syndrome (SIRS) is the concerted cytokine-driven response to an injury or an infection [3,4]. During the inflammatory response, the

*Address correspondence to this author at the New York Methodist Hospital, Brooklyn, New York, 232 Fitch's Pass, Trumbull, Connecticut 06611, USA; Tel: 1-203-261-8671; E-mail: plbern@yahoo.com liver responds by down regulation of transport protein production in order to produce higher levels of the acute phase proteins (APRs). C-reactive protein (CRP), named for its capacity to react with the $\mathrm{C}$-polysaccharide of $\mathrm{S}$. pneumonia, is the most important acute phase protein in its role in the inflammatory response and is elevated during inflammation as much as 50,000-fold to a peak in 48 hours [1]. Serum amyloid A is the only APR that is comparable to CRP in its sensitivity and rate of increase [1].

Plasma CRP is produced only by hepatocytes, predominantly under transcriptional control by the cytokine IL-6. The plasma half-life of CRP is constant under all conditions of health and disease and as such the only determinant of CRP concentration is the synthesis rate. The median concentration of CRP in young adult blood donors is $0.8 \mathrm{mg} / \mathrm{L}$, the $90^{\text {th }}$ percentile is $3.0 \mathrm{mg} / \mathrm{L}$, and the $99^{\text {th }}$ percentile is $10 \mathrm{mg} / \mathrm{l}$ [1]. The acute phase response drives the value from a hypothetical $5 \mu \mathrm{g} / \mathrm{l}$ to a value of $500 \mathrm{mg} / \mathrm{l}$ or more [1].

CRP is thought to act by physiologically binding with high affinity to phosphocholine residues as well as to ligands on foreign (or autologous) cellular, particulate or molecular structures leading to aggregation and activation of the classical complement pathway. A secondary effect of CRP is hypothesized in that its ligand binding property resembles the antibody response and in some circumstances may function as a proinflammatory mediator [5]. This process is mediated by interleukin 1 and 6 as well as tumor necrosis 
factor $(\mathrm{TNF} \alpha)$ resulting in an upregulation of the counterregulatory hormones (cortisol, glucagon and catecholamines) resulting in an acute concerted reaction with the production of energy with an increased metabolic rate associated with hyperthermia [3, 4]. This energy is consumed during the innate immune response for the mobilization of white blood cells and the attack and degradation of foreign cells.

The stepwise progression of the clinical response is SIRS ---> sepsis---> septicemia -----> septic shock ----> MOF (multiple organ failure) ----> death. SIRS patients, by definition, have two or more of the following criteria: Temperature $<36{ }^{\circ} \mathrm{C}$ (hypothermia) or Temperature $>38{ }^{\circ} \mathrm{C}$ (hyperthermia), Heart Rate $>90$ beats/minute, Respiratory Rate $>20$ breath/minute, Systolic Blood Pressure <90, white blood cell count $>12,000$ cells $/ \mathrm{mm}^{3}$ or $<4,000$ cells $/ \mathrm{mm}^{3}$. When two criteria are met SIRS is present $[6,7]$ and the patient may progress to sepsis but this often is not the case.

It may be difficult to distinguish a moderate state of disease such as pneumonia with SIRS from the severe and progressive state of sepsis, septicemia or even septic shock. CRP, a readily measured APR, may add information necessary to define the risk of patients thought to have SIRS and reduce type 1 classification errors (false positive) that arise with the use of the standard clinical criteria. The hypothesis of this study is that CRP is a laboratory parameter that can confirm the presence of SIRS with progression to early sepsis in conjunction with the established clinical parameters.

\section{MATERIALS AND METHODS}

\section{Patient Selection}

The Institutional Review Committee provided ethical approval for the study. The patients in the study were identified by a concurrent review of the hemogram data provided by the automated hematology counter combined with patient information in the electronic medical record. The laboratory parameters of the patients in the study were selected to have a high likelihood of correlation with systemic infection and were prospectively collected over a 4month period. The CRP, the absolute neutrophil count, the white blood cell count and the blood culture results were collected in all study patients. The ability to identify patients with SIRS associated with the progression to sepsis was the focus of the study. One hundred sixty eight patients with leukocytosis greater than 12,000 cells $/ \mathrm{mm}^{3}$ associated with a high absolute neutrophil count were studied. Although the ACCP definition of SIRS includes neutropenia as a component criterion such patients were not included in the analysis because of the potential confounding data that might have been provided by patients treated with anti-neoplastic chemotherapy. As the criteria stand: the criteria for selection were limited to presence of two of the following: body temperature $>38^{\circ} \mathrm{C}$ or $\left\langle 36^{\circ} \mathrm{C}\right.$, heart rate $>90$ beats $/ \mathrm{min}$, respiratory rate $>20 / \mathrm{min}$, white blood cell count $>12,000$ cells $/ \mathrm{mm}^{3}$, as well as more than $10 \%$ immature neutrophils. One hundred sixty eight patients met the inclusion criteria and were analyzed for CRP response.

\section{Inclusion Criteria}

The inclusion criteria were blood smears with WBC count $>12,000$ cells $/ \mathrm{mm}^{3}$ or absolute neutrophil count over
10,800 cells $/ \mathrm{mm}^{3}$ as well as smears with 10 percent immature neutrophils. CRP level, WBC count, percent neutrophils, age, gender, and date of sample were all recorded on Excel. CRP levels were assessed at the time of admission and the clinicians were blinded to the results. Systematic Inflammatory Response Syndrome (SIRS) is defined as a condition in which a patient displayed any two of the following abnormal vital signs $[6,7]$ :

\section{- $\quad$ Temperature $\left\langle 36{ }^{\circ} \mathrm{C}\right.$ (hypothermia) or Temperature $>$ $38^{\circ} \mathrm{C}$ (hyperthermia) \\ - Heart Rate $>90$ beats $/ \mathrm{min}$. \\ - Respiratory Rate $>20$ breath/min. \\ - $\quad$ Systolic Blood Pressure $<90 \mathrm{~mm} \mathrm{Hg}$ \\ - White blood cell count $>12,000$ cells $/ \mathrm{mm}^{3 *}$ \\ Exclusion Criteria}

Excluded were patients with normal blood smears or abnormalities due to primary blood diseases such as leukemia. *Our study did not use the selection criterion of < $4,000 \mathrm{wbc}$ cells $/ \mathrm{mm}^{3}$ for the reason stated above.

\section{Analytical Methods}

CRP was measured on the Roche Modular System, Roche Diagnostics, Indianapolis, Indiana. The hematology parameters were measured on the Coulter S Plus System, Beckman Coulter Inc., Brea, California.

\section{Reference Range for CRP}

The reference range for this limited SIRS study was determined by excluding cases that had bacteremia and also cases of pneumonia or pyelonephritis with the possibility that there might be seeding of bacteria into the circulation. It can't be called a reference range because of the considerartion pertaining to low level CRP and the work of Ridker and others, and the sample size and statistical variance in the unaffected patients studied for SIRS. The results are measured in $\mathrm{mg} / \mathrm{L}$. The CRP for an unaffected 135 patients had a geometric mean of $36.7 \mathrm{mg} / \mathrm{L}$ and a median of $47.6 \mathrm{mg} / \mathrm{L}$, with a nonparametric lower limit of $0.772 \mathrm{mg} / \mathrm{L}$ and a 90 percent confidence interval of 0.50 to $1.96 \mathrm{mg} / \mathrm{L}$. However, this can by no means be considered a reference population. One needs a non-hospitalized ambulatory population with perhaps a simulation model, and it would still not be sufficiently clean based on the Jupiter studies referred to in the introduction.

\section{Statistical Procedures}

All clinical features including absolute neutrophil count were scaled with a value of 0 denoting patients in the normal range, with a scale value of 1 denoting patients with abnormal clinical parameters. The CRP level was not included in the classification. A receiver operator characteristic (ROC) curve was constructed with a graphical plot of the sensitivity vs. (1- specificity) for a binary classifier system using several summed feature criteria from the definition of SIRS. This combination variable was plotted against CRP values to find the best sensitivity, specificity and area under the ROC curve (Fig. 1). Diagnoses and bacterial cultures were checked. The $\mathrm{X}^{2}$ values were calculated to determine the tests' significance. Statistical 
Table 1. Standard Descriptive Statistical Measures of Study Population

\begin{tabular}{|c|c|c|c|c|}
\hline & Mean & Std Dev* & Median & Geometric Mean \\
\hline \hline Age & 60.6 & 21.7 & 65 & 55.6 \\
\hline Total WBC** count & 20.2 & 17.7 & 16.5 & 17.5 \\
\hline Absolute neutrophils & 16.8 & 15.4 & 64.4 & 47.1 \\
\hline CRP & 105.3 & 110.8 & 98.5 & 95.6 \\
\hline Temperature & 98.6 & 1.4 & 85 & 85.5 \\
\hline Pulse & 87.3 & 17.2 & 20 & 20.4 \\
\hline Respiratory rate & 20.9 & 5.7 & & \\
\hline
\end{tabular}

*Standard deviation.

** white blood cell.

analyses were done on SPSS version 17, SPSS (version 18, IBM), Chicago, Illinois. ROC construction was done with MedCalc version 9.3.2.0, Frank Schoonjans, Belgium. Other critical procedures used were: One-way ANOVA, frequency tabulation, crosstable analyses by Chi-squared testing, Mann-Whitney test, and Kruskal-Wallis. Latent Class Models were explored using Latent Gold 4.0 (Statistical Innovations, Belmont, MA).

\section{Study Population Characteristics from Selection Criteria}

The description of the one hundred and sixty eight patient study population using the criteria described is shown in Table 1. There were 67 percent women and 33 percent men, and the median age was 65 years. Of the 168 patients in the study 95 had non-inflammatory diagnoses, 41 had SIRS or sepsis, and the remainder had neither a non-inflammatory diagnosis nor SIRS. Thirty percent of the population in the study were at high risk for sepsis, 79 percent had CRP over $50 \mathrm{mg} / \mathrm{L}, 49$ percent had CRP over 60, 7 percent had bacteremia (based on culture submissions), 33 percent had fever, 72 percent had tachycardia, 62 percent had elevated respiratory rate, and 84 percent had an absolute neutrophil count exceeding 12,000. The percent of the study patients with combined features increased is as follows: heart rate and respiration, 100\%; increased pulse, respiration and absolute neutrophil count, 67\%; increased pulse, respiratory rate, absolute neutrophil count and fever, 50\%. The remaining features are in Table $\mathbf{1}$ without further elaboration.

\section{RESULTS}

There were 35 positive blood cultures and 41 patients were diagnosed with sepsis based on clinical criteria and/ or blood cultures. A ROC curve showed an area under the curve (AUC) to be 0.777 and sensitivity over $75 \%$ at a CRP $=52 \mathrm{mg} / \mathrm{l}(95 \%$ confidence interval $63-84.7 \%)$ and sensitivity of $87.9 \%$ with a likelihood ratio positive of 1.88 at a critical value of 50. The ROC results are summarized in Table 2 and Fig. (1). The table indicates that at the chosen cutoff value for CRP, much higher than 20 or $30 \mathrm{mg} / \mathrm{l}$, the sensitivity is $75 \%$, and the specificity is $59 \%$. This indicates that at $52 \mathrm{mg} / \mathrm{l}$ cutoff, absence of serious infection is missed in almost 60 percent of cases. The Likelihood Ratio of a Positive result is $85 \%$ greater than 1 .
Table 2. The Results of the ROC Curve with CRP at $52 \mathrm{mg} / \mathrm{l}$

\begin{tabular}{|c|c|}
\hline ROC result & Combined Variable \\
\hline \hline Abs Neu & 10,800 \\
\hline Positive & 28 \\
\hline CRP & $52 \mathrm{mg} / \mathrm{L}$ \\
\hline AUC & 0.777 \\
\hline $95 \%$ CI & $0.569-0.800$ \\
\hline P & 0.0001 \\
\hline Sensitivity & 75 \\
\hline $95 \%$ CI & $63-84.7$ \\
\hline Specificity & 59.4 \\
\hline $95 \%$ CI & $49.2-69.1$ \\
\hline Likelihood Ratio Positive & 1.85 \\
\hline Positive Predictive Value & $54 \%$ \\
\hline
\end{tabular}

The table indicates that at the chosen cutoff value for CRP, much higher than 20 or 30 $\mathrm{mg} / \mathrm{l}$, the sensitivity is $75 \%$, and the specificity is $59 \%$. This indicates that at $52 \mathrm{mg} / \mathrm{l}$ cutoff, absence of serious infection is missed in almost 60 percent of cases. The Likelihood Ratio of a Positive result is $85 \%$ greater than 1 .

The absolute neutrophil count was not well correlated with the SIRS classification using binary combinations of temperature, pulse, respiratory rate and absolute neutrophil count to classify (Fig. 2), but there was a strong correlation of stepwise CRP increase with increased numbers of SIRS indicators shown in an error plot (Fig. 3). Fig. (3) has 3 means (and 95\% confidence intervals) with SIRS class $1(<2$ features) and SIRS class 3 (more than 2 features) clearly separated, which is t-test significant. ANOVA would be significant for the 3 groups. Table $\mathbf{3}$ shows the means and medians for the CRP in each of the linear combined feature groups. The results are significant across the classes. Even though the association between CRP and absolute neutrophil count is nonexistent $(\mathrm{R}=0.006$, there is an association between CRP with combined SIRS features giving greater diagnostic efficiency (Fig. 3) than with absolute neutrophil count (Fig. 2). 


\section{ROC Curve}

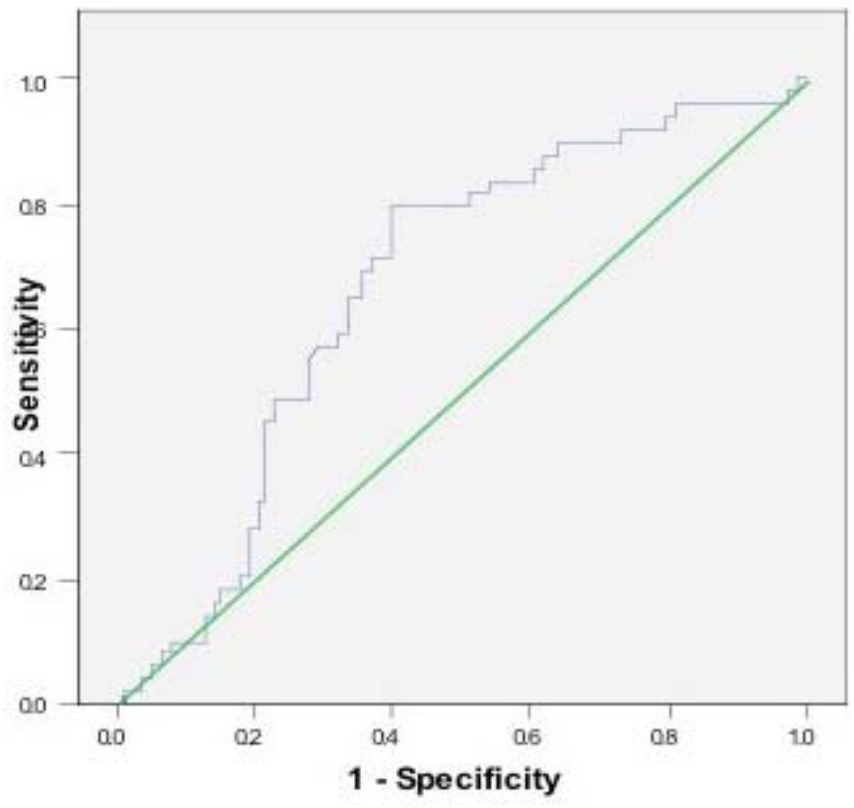

Diagonal segments are produced by fes.

Fig. (1). Receiver Operator curve for C-Reactive Protein in SIRS.

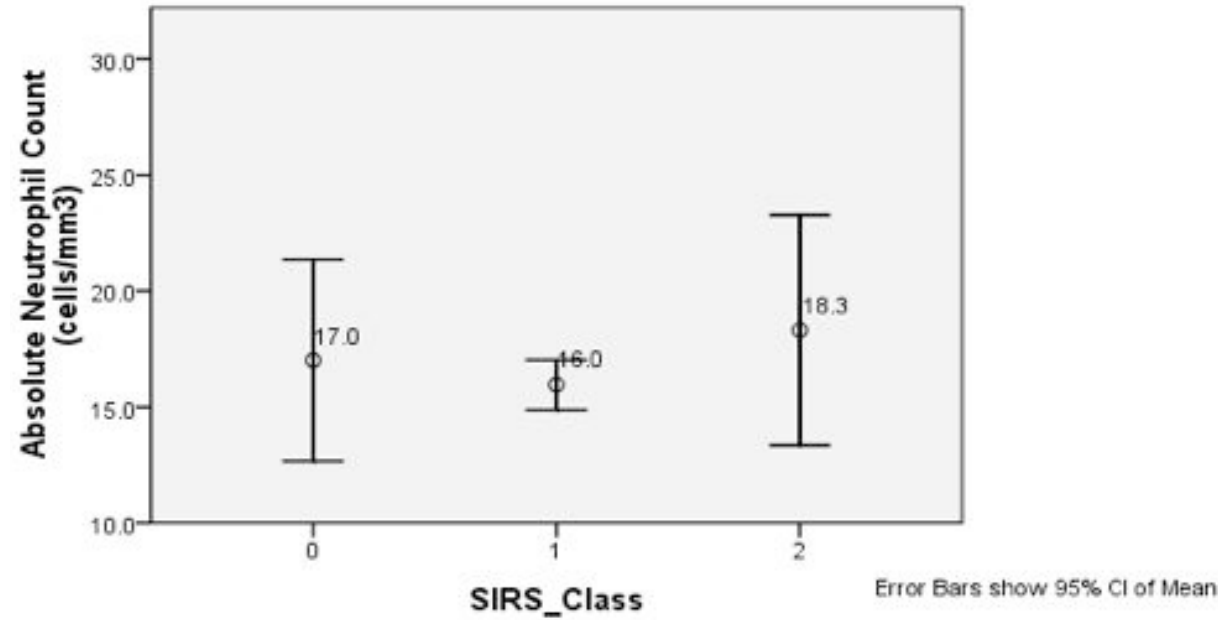

Fig. (2). Absolute Neutrophil Count by SIRS_Class by Sum of Features:

Absent Association between Absolute Neutrophil Count and SIRS.

SIRS Class derived from sum of features. Temperature, Pulse Rate, Respiratory Rate, and Absolute Neutrophil Count Value is 0 if sum is $<2$, 1 if 2 , and 2 if $>2$.

After the rescaling of CRP, CRP was combined with the defining variables for SIRS. A t-test was carried out on the CRP differences between the resulting low and high risk groups [2]. The results were significant at $p=0.0001$ with a Bonferroni test to correct for the unequal variance. Table 4 shows the t-test for CRP level vs the diagnosis of high risk for SIRS/sepsis or its absence. In this experiment CRP was excluded from the SIRS definition. There were 35 patients in the high-risk group, with a mean CRP at $152.71 \mathrm{mg} / \mathrm{L}$ vs 92 patients with a mean CRP at $94.65 \mathrm{mg} / \mathrm{L}$. The t-test compares the means and standard deviations of CRP between the 2 groups based on presence or absence of sepsis, establishing a significant relationship.

Chi-square analysis of these patients combined SIRS features (see Table 4) plus CRP for the diagnosis of sepsis was 17.2 , significant at $p=0.0001$. Table $\mathbf{5}$ shows that the association between combined SIRS features and sepsis is strong when CRP is elevated but weak when CRP is not elevated. The total number of patients is 127 . The sepsis positive patients is 35 with or without the CRP. But in the combined feature classes 2 and 3, there are 34 versus 29 


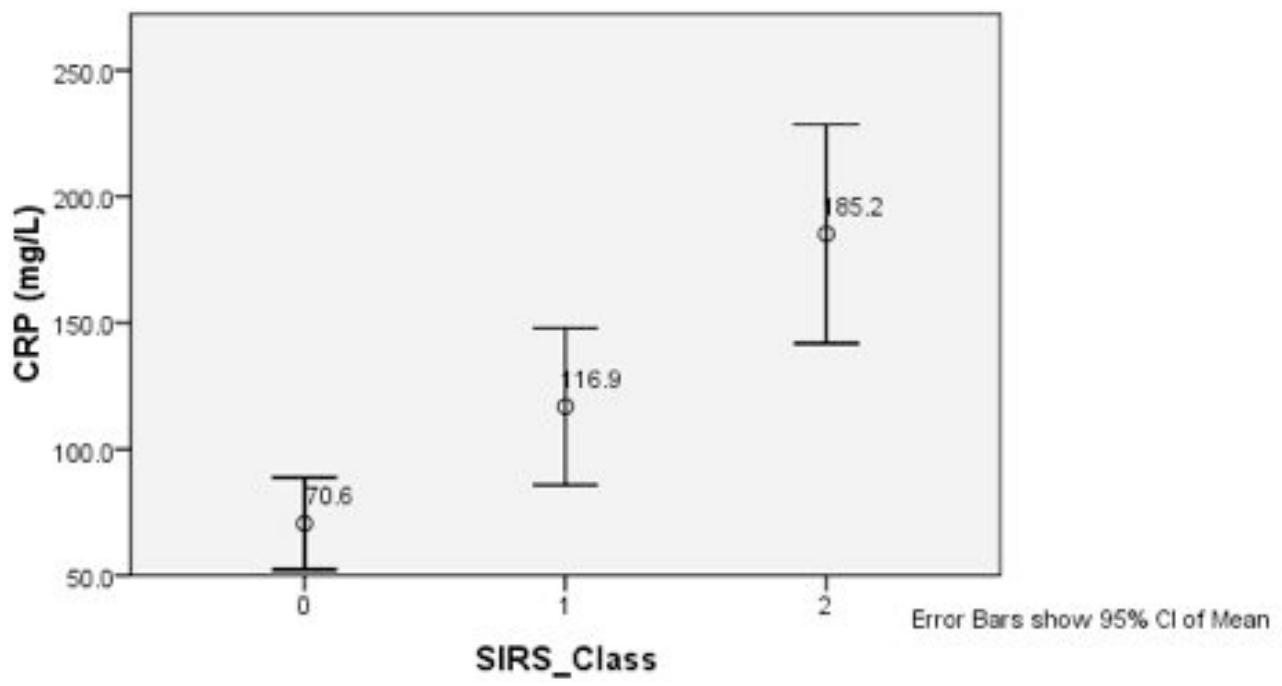

Fig. (3). C-Reactive Protein (CRP) by SIRS_Class by Sum of Features:

Association between CRP Level and SIRS_Class - with lower CRP with few features

SIRS Class derived from sum of features. Temperature, Pulse Rate, Respiratory Rate, and Absolute Neutrophil Count Value is 0 if sum is $<2$, 1 if 2 , and 2 if $>2$.

Table 3. Equality of Several Variances Test of CRP Grouped by Combination of by SIRS Features

\begin{tabular}{|c|c|c|c|}
\hline SIRS CRITERIA & N & Mean & Median \\
\hline \hline No SIRS features & 16 & 68.369 & 71.654 \\
\hline 1 SIRS feature & 67 & 115.763 & 77.700 \\
\hline 2 SIRS features & 57 & 176.692 & 19.500 \\
\hline 3 SIRS features & 25 & 254.433 & 166.500 \\
\hline 4 SIRS features & 3 & 157.300 \\
\hline
\end{tabular}

Table 3 shows the means and medians for the CRP in each of the linear combined feature groups. The ANOVA results are significant across the classes (N=168).

Table 4. t-test of Association for CRP and Diagnosis of Sepsis

\begin{tabular}{|c|c|c|c|c|}
\hline & \multicolumn{4}{|c|}{ Statistics for Diagnosis of Sepsis } \\
\hline Diagnosis & N & Mean (mg/l) & Standard Deviation & Standard Error \\
\hline \hline No sepsis & 92 & 94.65 & 107.62 & 11.22 \\
\hline Sepsis & 35 & 152.71 & 113.39 & 19.16 \\
\hline
\end{tabular}

$* \mathrm{P}<0.01$.

The t-test compares the means and standard deviations of CRP between two groups based on presence or absence of high risk SIRS or sepsis, establishing a significant relationship $(\mathrm{N}=127)$.

cases that are sepsis positive with CRP versus without adding CRP, a pickup of 5 cases in the highest risk group. There are 92 patients in the sepsis negative group, with or without CRP. In the feature class of $2+3,68$ versus 59 cases are seen with and without CRP. This is 9 fewer negative for sepsis cases without CRP (false positive CRPs). On the other hand, in the low feature class 1 ( $<2$ features) there are 24 versus 33 with versus without CRP, a 9 case advantage. The $\mathrm{N}$-by-N table allows for chi-squared testing several ways, and also for calculation of sensitivity and specificity of sepsis detection with the compared combinations (you can do the math!). The results are significant for the addition of
CRP. Table 6 shows the means for the CRP combined with SIRS features. If the groups with 2 and 3 SIRS criteria are combined, the significance by ANOVA or by Spearman rank correlation (Spearman's rho) (The two sets of variables are ranked separately and the differences in rank, $d$, are calculated for each pair of variables), would be significant.

We present another series of experiments that (a) depicts the difficulties of interpreting the standard hemogram for recognizing SIRS and distinguishing moderate from severe SIRS, and (b) highlights the value of CRP in identifying significant SIRS despite its shortcomings as a univariate 
Table 5. Chi-Square Tests of Association for Combined Feature Class with and without CRP and Sepsis Diagnosis

\begin{tabular}{|c|c|c|c|c|c|}
\hline Feature Class & & \multicolumn{2}{|c|}{ Sepsis Diagnosis } & Polumn sum \\
\hline \hline & & Negative & Positive & 25 \\
\hline With CRP & 1 & 24 & 1 & 44 & 58 \\
\hline & 2 & 34 & 24 & 10 & 39 \\
\hline Without CRP & Row sum & 34 & 92 & 6.0001 \\
\hline & 2 & 33 & 18 & 61 \\
\hline
\end{tabular}

Table 5 is an N-by-N construction for the chi-square test for the significance of associations between adding CRP to the feature class(es) of combinations thereof. Feature classes from 1-3 are: 1, 1 or fewer SIRS features; 2,2 features; 3,3 or 4 features. The features consist of temperature, pulse, respiratory rate, and absolute

neutrophil count. In combined $2+3$, there is a pickup of 5 cases in the highest risk group by adding CRP. In the lowest class group 1, there is a 9 case advantage with added CRP

Table 6. Equality of Several Variances Test of CRP Grouped by SIRS Features and CRP $(p<0.0001)$

\begin{tabular}{|c|c|c|c|}
\hline GROUP & N & Mean (mg/L) & Median (mg/L) \\
\hline \hline CRP, but no SIRS features & 10 & 713.9 & 71 \\
\hline CRP + 1 SIRS feature & 53 & 122.5 & 173.1 \\
\hline CRP + 2 SIRS features & 42 & $\underline{254.4}$ & 165.4 \\
\hline CRP + 3 SIRS features & 19 & $\underline{3}$ & 157.3 \\
\hline CRP + 4 features & 22 & 127 & $78.9)$ \\
\hline CRP + 3,4 SIRS features & Column total & & \\
\hline
\end{tabular}

The stepwise increase in CRP means and medians with CRP added to classes with $0-4$ SIRS features. Classes with 3,4 features have been combined so that no cell $\mathrm{N}$ has fewer than 3 cases.

Table 7. One-Way Anova for WBC Count, Neutrophil/tot WBC Count, CRP and High Risk for Severe SIRS

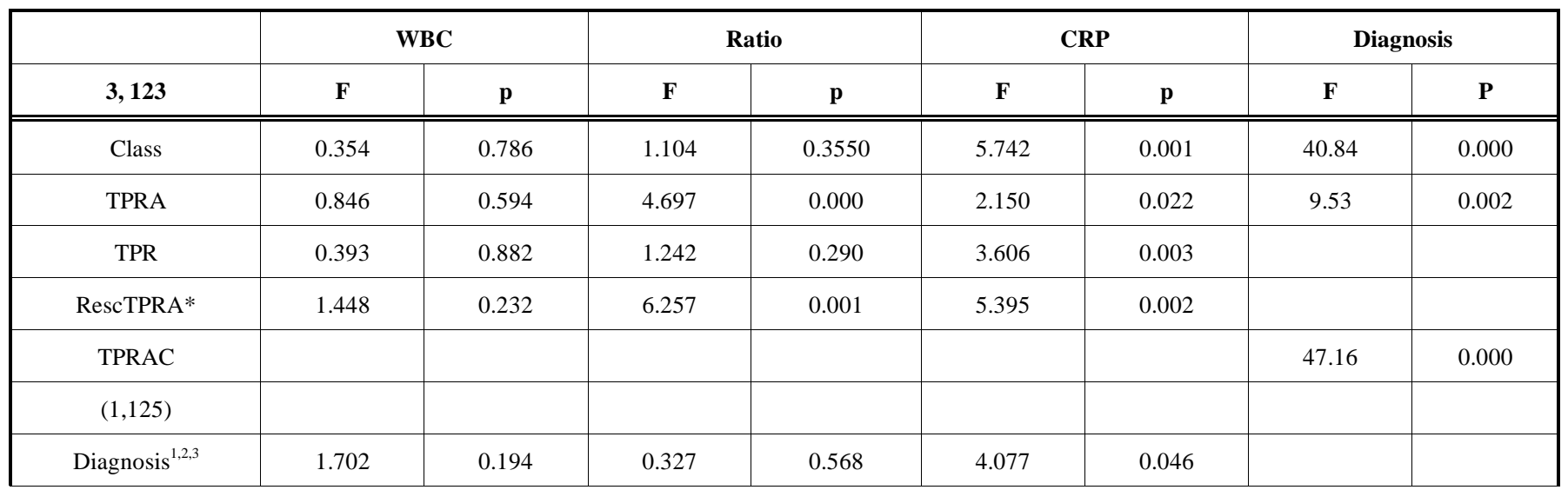

${ }^{1}$ Temp [F] 3.711, [p] 0.056 .

${ }^{2}$ Pulse $[\mathrm{F}] 14.732$, [p] 0.000

${ }^{3} \operatorname{Resp}[\mathrm{F}] 2.193,[\mathrm{p}] 0.141$

*RescTPRA: rescaled TPRA, where A is absolute neutrophil count.

This Table (7) is notable for the following: (1) Class, TPRA, and TPRAC predict diagnosis; There is no association with WBC count and any of the classifications based on differences in means and confidence intervals. There is an association between both TPRA and RescTPRA based upon ratio of absolute neutrophil count and total WBC. CRP means and $95 \%$ confidence intervals differ significantly among class, TPRA, TPR, and rescaled TPRA subsets. 
predictor; (c) compares classifications that use the strength of variable combinations and gives validation to the classical SIRS criteria. In the first experiment (Table 7) we produce a one-way ANOVA of the means (and variances) comparing the WBC count, percent neutrophil count, CRP, and diagnosis of SIRS, high risk for sepsis, among subclasses of class, TPRA, TPR alone, a rescaled TPRA, and diagnosis ( 0 or 1) using the127 patient complete data set and the feature combinations used to classify. The ANOVA is not optimal when the distribution of the variables within the classification is not normal. Table $\mathbf{8}$ is a crosstables analysis of the classifications by diagnosis of high risk SIRS. The measures shown are Chi-squared $\left(\mathrm{X}^{2}\right)$, significance $(\mathrm{p})$, likelihood ratio (LR), Phi, and Kruskal-Wallis test (KW). The validity of using the feature combinations is supported. Table 9 is a well validated 2-class ordinal regression Latent Class Analysis with all Chi-squared measures at $\mathrm{p}=1$.

A 4-class Latent Class Cluster Model had a model $\mathrm{L}^{2}=$ 75.2. The improvement in $\mathrm{L}^{2}$ is attributable to the cluster model vs the ordinal regression model.

\section{DISCUSSION}

CRP adds information necessary to assess the risk of patients thought to have SIRS and reduces type-1 classification errors (false positives). The best indicator of SIRS is a combination of clinical and laboratory parameters. Defining criteria include fever, tachypnea, tachycardia, and leukocytosis with neutrophilia [6, 7]. It may be difficult, however, to distinguish the moderate state of the disease such as pneumonia with SIRS from the severe and progressive state of sepsis, septicemia or even septic shock [8-10]. When the SIRS criteria are used in clinical practice there appears to be a significantly high false positive rate. This results in a conundrum in that it is unlikely that a patient with sepsis would be missed, which is accepted, but it puts a heavy burden on the triage process and heavy reliance on unspecified clinical skills for the decision to admit or discharge with medication. The reverse side of the coin is that patients who are admitted and later are identified with bacteremia may not be reimbursed for the sufficient degree of severity of disease.

CRP may be a sensitive indicator that can confirm the presence of early sepsis in conjunction with the established clinical parameters. In clinical practice SIRS is often equated with sepsis, with or without associated infection. When SIRS is unchecked a progressive clinical response culminating in septic shock, multiple organ failure and death may be seen. If SIRS can be identified at an early point then goal directed therapy, within best practice guidelines, can be instituted in a more aggressive fashion.

Patients with increasing numbers of SIRS criteria in conjunction with CRP level measurement were studied. Although the variance of CRP was found to be too broad to differentiate between moderate and severe sepsis, CRP was found to be sensitive for ruling in for sepsis when correlated with the combined SIRS criteria. As such CRP is a sensitive indicator that can confirm the presence of early sepsis in conjunction with the established clinical parameters thus reducing the likelihood of type-1 error (false positive) and providing greater diagnostic efficiency.

\section{Significant Issues that Might be Raised Concerning the Methodology Results}

The patient selection was confined to patients with white blood cell count over $12,000 / \mathrm{mm}^{3}$ for reasons stated in the methods section. All of the specimens were personally selected by the pathologist in charge of the clinical laboratory by identifying the specimens reviewed for abnormal morphology daily, and the review of the daily archives of specimens for hemogram analysis. Pooled specimens were run twice daily. The study was unfunded and the resources to conduct a more controlled study were unavailable, except for the enthusiastic work by volunteer honors biology students. Insofar as the reference range for CRP is concerned, there is an epidemiological range that is irrelevant for the study, which is not in any way concerned with cardiovascular risk factors. The authors reject a definition of the reference range based on the value below which there is no SIRS and above which there is SIRS. Having extensive experience with laboratory outcomes research, the interest here is to find a probabilistic model that predicts the risk of developing SIRS evolving to sepsis and multi-organ failure with minimum error, closing the huge false positive gap in the reliance on SIRS criteria. The gap is even partly created by assigning unweighted values to the observations used in assessing the disease state. In this respect, one has to ask the question how there can be a useful reference range for a cytokine driven inflammatory marker that may be different for obesity and type 2 diabetes, perhaps related to cardiovascular or stroke risk, and a different range for pneumonia/ pyelonephritis, and still another for bacteremia? The criteria found from the complete data set are optimal for the entropy of the data.

Alternative markers may, in the future, supplant CRP in order to provide additional diagnostic efficiency. Procalcitonin (PCT), a prohormone of calcitonin, is a potential alternative for CRP, and it may be used for differentiation between moderate and severe sepsis. Procalcitonin is almost undetectable under physiological conditions ( $\mathrm{pg} / \mathrm{ml}$ range), but rises to considerably higher values within 4-6 hours in response to bacteremia, and appears to be related to the severity of infection [11]. An immunoluminometric procalcitonin assay is now commercially available (BRAHMS Diagnostika, Berlin, Germany) and values can be obtained on a routine basis within two hours of blood sampling. At the time of the present study PCT was not, (and at present still is not), generally available in North American clinical laboratories.

The usefulness of having CRP with transthyretin (TTR) and albumin has been noted [12-16], but that was not the focus of our study. The reference ranges for albumin, transferrin, and TTR, respectively, are usually cited as: 35$50 \mathrm{~g} / \mathrm{L}, 1980-3270 \mathrm{mg} / \mathrm{L}$, and $190-350 \mathrm{mg} / \mathrm{L}$. It is of note that energy consumption as well as production of CRP is directly affected by protein reserve as measured by those markers that reflect the balance of protein synthesis and degradation such as transthyretin (TTR). While the so called negative indicators of inflammation are not the focus of this study, the reprioritization of protein synthesis by the liver results in increased CRP and $\alpha 1$ acid glycopotein, while the transthyretin, albumin, and transferrin synthesis are blunted. At the same time, skeletal muscle provides gluconeogenic 
Table 8. Crosstables Analysis of Classified Data by Diagnosis of High Risk Sirs

\begin{tabular}{|c|c|c|c|c|c|c|c|}
\hline & CHISQ & p & LR & Phi & p & KW* & \\
\hline \hline DIAGNOSIS & & & & & & \\
\hline TPRAC & 36.87 & 0.000 & 42.19 & 0.539 & 0.000 & 35.86 \\
\hline TPRA & 35.44 & 0.000 & 38.85 & 0.528 & 0.000 & 30.40 & 0.000 \\
\hline TPR & 35.33 & 0.000 & 35.35 & 0.512 & 0.000 & 31.92 \\
\hline CLASS & 32.25 & 0.000 & 36.33 & 0.504 & 0.000 & 0.000 \\
\hline RESC_TPRA & 31.24 & 0.000 & 33.05 & 0.496 & 0.000 & \\
\hline CLASS & & & & & & \\
\hline RESC_TPRA & 254.93 & 0.000 & 258.93 & 1.417 & 0.000 & \\
\hline TPRAC & & & & & & \\
\hline TPR & 250.49 & 0.000 & 195.82 & 1.404 & 0.000 & \\
\hline
\end{tabular}

*KW: Kruskal-Wallis test.

Table $\mathbf{8}$ is a nonparametric (normality is not assumed) association between (1) TPRAC, TPRA, TPR, class and rescaled TPRA by rank order within subsets in predicting diagnosis; (2) rescaled TPRA in predicting class; (3) TPR in predicting TPRAC. These associations are all significant at less than 0.0001 . It is interesting that TPR is strongly correlated with TPR[AC], TPR with absolute neutrophil count and CRP in a crosstable analysis.

Table 9. 2-Class Ordinal Regression Model by TPRAC And CRP With High Risk Diagnosis as Covariate

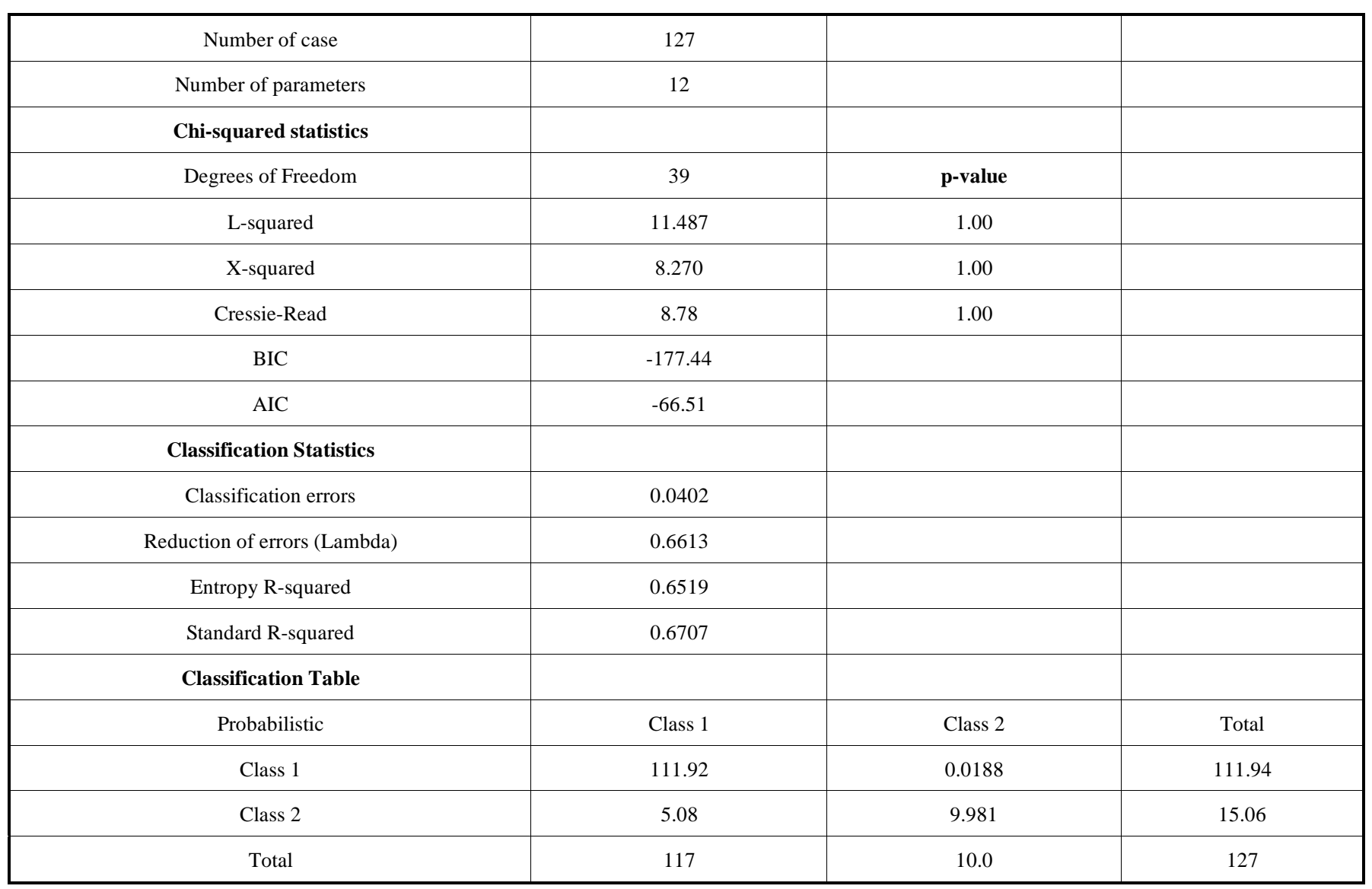

Table $\mathbf{X}$ is a 2-class ordinal regression model to predict high risk SIRS absent or present using TPRAC and CRP as predictors. The model is validated for goodness of fit by $\mathrm{L}^{2}, \mathrm{X}^{2}$, and Cressie-Read all at p-value $=1$. There is a 4\% classification error. BIC and AIC are entropy measures and refer to Baye's Information Criterion, and to Akaike's Information Criterion. AIC is an improvement on the work of Solomon Kullback, and received a distinguished award for Japanese discovery.

precursors to the bloodstream, as glycogen stores in liver become depleted. Although the response may initially promote survival of the organism it may become counterproductive over time. As such the use of CRP as a biomarker represents a potential real-time improvement in clinical practice even though it may be supplanted in the 
future. Further, the concept of combining CRP with a negative indicator of the inflammatory response, such as TTR, should be seriously considered.

Although CRP has been found in this study to be a sensitive marker for early SIRS with progression to sepsis, the use of PCT has promise to be a superior method for identifying moderate to severe sepsis and may supplant CRP. Both markers may facilitate the wider institution of best practice guidelines [17-22].

\section{CONCLUSION}

CRP indeed has validity for confirming the presence of SIRS with progression to sepsis even when clinical features are incomplete or equivocal.

\section{CONFLICT OF INTEREST}

No funding or support was provided by any organization. None of the authors is or has been employed by any organization that may gain or lose financially from the results or conclusions of the study. None of the authors have any conflicting interests.

\section{ABBREVIATIONS}

$$
\begin{aligned}
& \text { APR }=\text { acute phase reactants } \\
& \text { CRP }=\text { C-reactive protein } \\
& \text { SIRS }=\text { systemic inflammatory response syndrome } \\
& \text { MOF }=\text { multiple organ failure } \\
& \text { WBC }=\text { white blood count } \\
& \text { ROC }=\text { receiver operating curve } \\
& \text { PCT }=\text { procalcitonin }
\end{aligned}
$$

\section{REFERENCES}

[1] Pepys MB, Hirschfield GM. C-reactive protein: a critical update. J Clin Invest 2003; 111: 1805-12.

[2] Despre JP. Bringing Jupiter down to earth. Lancet 2009; 373: 114748

[3] Ingenbleek $\mathrm{Y}$, Bernstein LH. The stressful condition as a nutritionally dependent adaptive dichotomy. Nutrition 1999; 15: 305-20.

[4] Ingenbleek Y, Bernstein LH. The nutritionally dependent adaptive dichotomy (NDAD) and stress hypermetabolism. J Clin Ligand Assay 1999; 22: 259-67.

[5] Briles DE, Forman C, Horowitz JC, et al. Antipneumococcal effects of C-reactive protein and monoclonal antibodies to pneumococcal cell wall and capsular antigens. Infect Immun 1989; 5: $1457-64$.
American College of Chest Physicians/Society of Critical Care Medicine. Consensus Conference: definitions for sepsis and organ failure and guidelines for the use of innovative therapies in sepsis. Crit Care Med 1992; 20(6): 864-74.

[7] American College of Chest Physicians/Society of Critical Care Medicine. Definitions for sepsis and organ failure and guidelines for the use of innovative therapies in Physicians/Society of Critical Care Medicine. Chest 1992; 101: 1644-55.

[8] Dupond JL, de Wazieres B, Million P, Humbert P, Gibey R Neutrophilic leukocytosis of systemic or bacterial origin: discriminative C-reactive protein? Rev Med Intern 1990; 11: 28992.

[9] Chi C, Shiesh S, Chen K, WU M, Lin X. C-reactive protein for the evaluation of acute abdominal pain. Am J Emerg Med 1996; 14: 254-6.

[10] Ishida H, Ichihara K, Matsuda N. Exploratory analysis of elevated C-reactive protein without leukocytosis from the clinical laboratory database. Jpn J Clin Pathol 2000; 48: 722-9.

[11] Simon L, Gauvin F, Amre DK, Saint-Louis P, Lacroix J. Serum procalcitonin and C-reactive protein levels as markers of bacterial infection: a systematic review and meta-analysis. Clin Infect Dis 2005; 39: 206-17.

[12] Ingenbleek Y. Why should plasma transthyretin become a routine screening tool in elderly persons? Editorial. J Nutr Health Aging 2009; 13(7): 640-2.

[13] Ingenbleek Y, Young VR. Significance of transthyretin in protein metabolism. Clin Chem Lab Med 2002; 40(12): 1281-91.

[14] Bernstein LH, Ingenbleek Y. Transthyretin: its response to malnutrition and stress injury. Clinical usefulness and economic implications. Clin Chem Lab Med 2002; 40: 1344-8.

[15] Bernstein, LH. Transthyretin and the systemic inflammatory response. Curr Nut Food Sci 2009; 5: 71-4.

[16] Devakonda A, George L, Raoof S, et al. Transthyretin as a marker to predict outcome in critically ill patients. Clin Biochem 2008; 41 : 1126-30.

[17] Arkader R, Troster EJ, Lopes MR, et al. Procalcitonin does discriminate between sepsis and systemic inflammatory response syndrome. Arch Dis Child 2006; 91: 117-20.

[18] Castelli GP, Pognani C, Meisner M, et al. Procalcitonin and Creactive protein during systemic inflammatory response syndrome, sepsis and organ dysfunction. Crit Care 2004; 8: R234-R42.

[19] Kofoed K, Andersen O, Kronborg G, et al. Use of plasma Creactive protein, procalcitonin, neutrophils, macrophage migration inhibitory factor, soluble urokinase-type plasminogen activator receptor, and soluble triggering receptor expressed on myeloid cells-1 in combination to diagnose infections: a prospective study. Crit Care 2007; 11: R38.

[20] Indino P, Lemarchand P, Bady P, et al. Prospective study on procalcitonin and other systemic infection markers in patients with leukocytosis. Int J Infect Dis 2008; 12: 319-24.

[21] Dandona P, Nix D, Wilson MF, et al. Procalcitonin increase after endotoxin injection in normal subjects. J Clin Endocrinol Metab 1994; 79: 1605-8.

[22] Bernstein LH, Devakonda A, Engelman E, Pancer G, Ferrara J. The role of procalcitonin in the diagnosis of sepsis and patient assignment to medical. J Clin Ligand Assay 2007; 30: 98-104. 